\title{
Underwater flexible mechanoreceptors constructed by anti-swelling self-healable hydrogel
}

\author{
Xin Liu ${ }^{1,2}$, Qin Zhang ${ }^{1}$, Fei Jia ${ }^{1^{*}}$ and Guanghui Gao ${ }^{1,2^{*}}$
}

\begin{abstract}
Wet-resistant flexible electronics have acquired increasing attention on applications in wet environments, such as sweaty skin, rainy weather, biological fluids, and underwater. However, it remains challenging to achieve nonswelling and underwater self-healing hydrogel sensors for the mechanical perception in aqueous solutions. Herein, a selfhealing and non-swellable hydrogel is successfully fabricated, which presents an automatically healing behavior in various aquatic environments, including deionized water, seawater, sweat, alkali and acidic aqueous solutions. Moreover, the hydrogel demonstrates high stretchability and stable electromechanical sensing properties in water. Furthermore, an electronic skin is designed with the features of fast responsiveness, reliability, and high sensitivity for detecting breathing, speaking, coughing, and diverse body movements. The self-healing hydrogel sensors enable a brilliant mechanical sensibility for detecting a series of dynamic stimuli in air and underwater, even after the healing of fracture interface in water. The underwater self-healing and anti-swelling hydrogel would provide enticing potential on various stable electronic devices for aquatic environments, such as implantable electrodes, triboelectric nanogenerators, and underwater soft robotics.
\end{abstract}

Keywords: hydrogel, water-insensitive electronics, underwater self-healing, anti-swelling, underwater sensing

\section{INTRODUCTION}

Flexible electronics have aroused infinite possibilities for the era of the internet of things [1], such as implantable bioelectronics [2,3], human-machine interface [4,5], electronic skins $[6,7]$, wearable sensors $[8,9]$, soft machines $[10,11]$, and energy storage devices [12,13]. Hydrogel electronics are greatly desirable for versatile human-machine interface, based on the advantage of tissue-similarity, biocompatibility, mechanical adaptability, and skin-like modulus [14]. In order to optimize the practical applications of hydrogel electronics, tremendous efforts have been devoted to constructing various hydrogel electronics with different promising functions, such as self-adhesive [15], temperature-tolerant [16], self-healing [17], and non-swelling [18]. Among them, underwater hydrogel electronics have drawn much interest in implantable electronics, brain electrodes, and underwater monitoring. Therefore, constructing nonswelling and underwater self-healing hydrogels is critical for the promising applications of flexible electronics in wet or aquatic environments.

However, it remains challenging to simultaneously integrating underwater self-healing and non-swellable properties into one hydrogel system $[19,20]$. Firstly, the aquatic environments would enable hydrogels an obvious swelling behavior with loss of initial network structure and function [21,22]. Besides, water molecules would saturate and interact with "active sites" of the healing interface via the acceptor and donor of hydrogen bonding, which would further cause the hydrolytic degradation of damaged surfaces [23-25]. Recently, some self-healing flexible electronics in wet environments have been reported, but the existing self-healing electronics were mostly constructed by the rigid and modulus-mismatch elastomer system [26-28]. Although various strategies have been innovatively developed, it remains challenging to fabricate underwater-healable and non-swellable hydrogel electronics to promote stable and reliable electronic applications in wet or aquatic environments. In addition, the non-swelling property of hydrogels tends to require a high degree of crosslinking to resist swelling stress, but highly cross-linked networks inevitably limit the occurrence of self-healing of hydrogels $[21,29]$. Ac-

${ }^{1}$ Polymeric and Soft Materials Laboratory, School of Chemical Engineering and Advanced Institute of Materials Science, Changchun University of Technology, Changchun 130000, China

2 Beijing Graphene Institute (BGI), Beijing 100095, China

* Corresponding authors (emails: jiafei@ccut.edu.cn (Jia F); ghgao@ccut.edu.cn (Gao G)) 
cordingly, a practical design of molecular network structure is a prerequisite for constructing self-healing hydrogel electronics for underwater applications.

In nature, the globular protein consisting of a hydrophilic shell and hydrophobic nucleus can quickly and effectively repair its structure and function of damage from the high hydrostatic pressure or accidental cutting, based on the synergistically hydrophilic and hydrophobic structure of the protein [30-32]. Inspired by its repairing mechanism, it is envisioned that skillfully designing and constructing the hydrophilic and hydrophobic network structure would provide a promising possibility of achieving underwater self-healing of water-rich soft materials.

In this work, methacrylamide (MAm) was selected to construct the polymer network of hydrogel, based on its inherent advantage of appropriate hydrophobicity from the methyl group and hydrophilicity of the amide group. Moreover, graphene (Gr) was introduced to endow hydrogels with robust mechanical properties and stable conductivity for electronic skins. The hydrophobicity of the supramolecular network could effectively repel water molecules and generate the hydrophobic interaction at the re-contactable fracture interface, and then the molecular permeation and hydrogen bonding will be further activated to construct the underwater self-healing hydrogels. Furthermore, a water-insensitive self-healing hydrogel sensor was successfully fabricated and exhibited an outstanding sensitivity for monitoring breathing, speaking, coughing, and diverse body movements even after autonomous healing in air or water. The water-insensitive self-healing electronics would enable an enticing inspiration for developing the next-generation flexible electronics, such as bioelectronics, electronic skins, human-machine interfaces, and implantable devices.

\section{EXPERIMENTAL SECTION}

\section{Materials}

Gr containing ethyl alcohol (0.5 wt\%) was prepared in Yun Shan Technology (Weihai, China) (TTWL2-03). MAm (99.5\%), and potassium persulfate (99\%, KPS) were supplied by Aladdin Reagent Co. (Shanghai, China). The $\mathrm{KCl}, \mathrm{MgCl}_{2}, \mathrm{MgSO}_{4}, \mathrm{NaCl}$, and $\mathrm{CaCl}_{2}$ were obtained from the Tiantai Chemical Works Company (Tianjin, China). Deionized water was prepared through a water purifier in the laboratory, and the resistivity of water was $18.2 \mathrm{M} \Omega \mathrm{cm}$. One liter of seawater with $3.44 \%$ salinity was prepared from $7 \mathrm{wt} \% \mathrm{KCl}, 2.3 \mathrm{wt} \% \mathrm{MgCl}_{2}, 3.2 \mathrm{wt} \%$ $\mathrm{MgSO}_{4}, 26.7 \mathrm{wt} \% \mathrm{NaCl}$ and $1.15 \mathrm{wt} \% \mathrm{CaCl}_{2}$. The $1 \mathrm{~L}$ sweat consists of $0.5 \mathrm{wt} \% \mathrm{NaCl}, 0.1 \mathrm{wt} \%$ lactic acids, and $0.1 \mathrm{wt} \%$ urea, and the $\mathrm{pH}$ of sweat was adjusted to 6.5. Hydrochloric acid and sodium hydroxide were used to adjust the $\mathrm{pH}$ of aqueous solutions ( $\mathrm{pH} 3$ and 11).

\section{Preparation of self-healing hydrogel}

Firstly, the homogeneous Gr solution consisting of $\mathrm{Gr}$ $(0.2 \mathrm{~g})$ and water $(10 \mathrm{~mL})$ was prepared through the ultrasound treatment for $1 \mathrm{~h}$. Next, the monomer of MAm $(2.8,3.0,3.2$, or $3.4 \mathrm{~g})$ and initiator of KPS $(0.04 \mathrm{~g})$ were added into the as-prepared $\mathrm{Gr}$ solution and continuously stirred at $40^{\circ} \mathrm{C}$ until they were completely dissolved. Finally, the self-healing hydrogel was prepared through the copolymerization of MAm in the reaction mold at $45^{\circ} \mathrm{C}$ for $12 \mathrm{~h}$. The hydrogel was defined as Gr-PMAm-2.8/3.0/ 3.2/3.4.

\section{Preparation of polyacrylamide hydrogel}

The polyacrylamide (PAm) hydrogel was prepared through the same method. In brief, the homogeneous Gr solution consisting of $\mathrm{Gr}(0.2 \mathrm{~g})$ and water $(10 \mathrm{~mL})$ was firstly prepared. Next, the monomer of acrylamide (Am) $(3.4 \mathrm{~g})$ and initiator of KPS $(0.04 \mathrm{~g})$ were added into the as-prepared $\mathrm{Gr}$ solution and continuously stirred at $40^{\circ} \mathrm{C}$ until they are completely dissolved. Finally, the PAm hydrogel was prepared in the reaction mold at $70^{\circ} \mathrm{C}$ for $12 \mathrm{~h}$. The hydrogel was defined as Gr-PAm.

\section{Mechanical properties}

Mechanical tensile properties of the hydrogel samples were determined by a stretching machine with a crosshead tester (AGS-X-100 N, SHIMADZU) with a stretching rate of $100 \mathrm{~mm} \mathrm{~min}^{-1}$. The hydrogel samples were cut into the dumbbell shape before testing $(30 \mathrm{~mm} \times$ $2 \mathrm{~mm} \times 4 \mathrm{~mm}$ ). Each hydrogel sample was tested repeatedly three times.

\section{Swelling behavior}

The swelling performance of PMAm and PAm hydrogels was studied by soaking of the samples. First, the initial weight of hydrogels was weighed and defined as $W_{0}$. The hydrogels with equivalent specification were respectively immersed in various aqueous solutions, and the weight of samples was weighed after some time and recorded as $W_{\mathrm{i}}$. The swelling ratio (SR) was calculated through $\left(W_{\mathrm{i}}-\right.$ $\left.W_{0}\right) / \mathrm{W}_{0}$ to evaluate the swelling capacity of hydrogels.

\section{Rheological measurement}

Rheometer (MCR-302, Anton Paar) was used to measure the viscoelasticity of hydrogels. The strain sweep $(\gamma)$ was 
set to a range from $0.01 \%$ to $1000 \%$. Viscoelasticity recovery of hydrogels was evaluated at the strain between $50 \%$ and $0.1 \%$ and a frequency of $10 \mathrm{rad} \mathrm{s}^{-1}$ at $25^{\circ} \mathrm{C}$.

\section{Electrical resistance measurement}

The resistance variation of hydrogels was measured using a PAR-2273 electrochemical station by linking the hydrogel samples to the testing system. The initial resistance and real-time resistance of samples were recorded as $R_{0}$ and $R_{\mathrm{i}}$, respectively, and $\Delta R$ was calculated by $R_{\mathrm{i}}-R_{0}$.

\section{Thermogravimetric analysis}

The thermogravimetric analysis (TGA) was performed by the Pyris1 TGA (Perkin Elmer). The hydrogel samples were heated from 30 to $700^{\circ} \mathrm{C}$ at a heating rate of $10^{\circ} \mathrm{C} \mathrm{m^{-1 }}$ in a nitrogen atmosphere.

\section{RESULTS AND DISCUSSION}

We successfully designed an underwater self-healing hydrogel conductor for underwater mechanoreceptors. The self-healing hydrogel was manufactured via the polymerization of MAm in an aqueous solution rich in Gr. The fabrication process of the self-healing hydrogel is illustrated in Fig. 1. Gr was firstly added into water to prepare a suspension, and then MAm and KPS were further dissolved in the solution. Finally, the underwater self-healing hydrogels were fabricated through thermalinitiation polymerization. The methyl and amide groups of polyMAm (PMAm) were evenly distributed in the polymer chain, which endowed the hydrogels with synergistically hydrophobic and hydrophilic networks for water-resistance, while the introduction of Gr enabled the robust mechanical property and excellent conductivity of hydrogels. The effect of Gr on the microstructure of hydrogels was demonstrated by the scanning electron microscopy in Fig. S1 which shows a significantly regulated network structure.

The underlying underwater self-healing mechanism was further discussed in Fig. S2. When hydrogel was damaged in the aquatic environments, the water molecule instantaneously filled the whole fracture interface of hydrogels, which seriously hindered the emergence of healing interaction. However, once two fractured segments of hydrogels were subjected to a slight pressure to re-contact, the hydrophobicity of the methyl group in the fractured interface would effectively resist and repel the interfacial water and further generate strongly waterresistant hydrophobic interaction to activate the healing. Furthermore, the hydrophobic interaction, intermolecular hydrogen bonding, and kinematic entanglement of polymer chains would be further formed, thus enhancing the healing effects. Accordingly, the hydrogel presented a prominent underwater self-healing performance.

The swelling behavior is a crucial parameter to investigate the water-resistance of hydrogel applied in

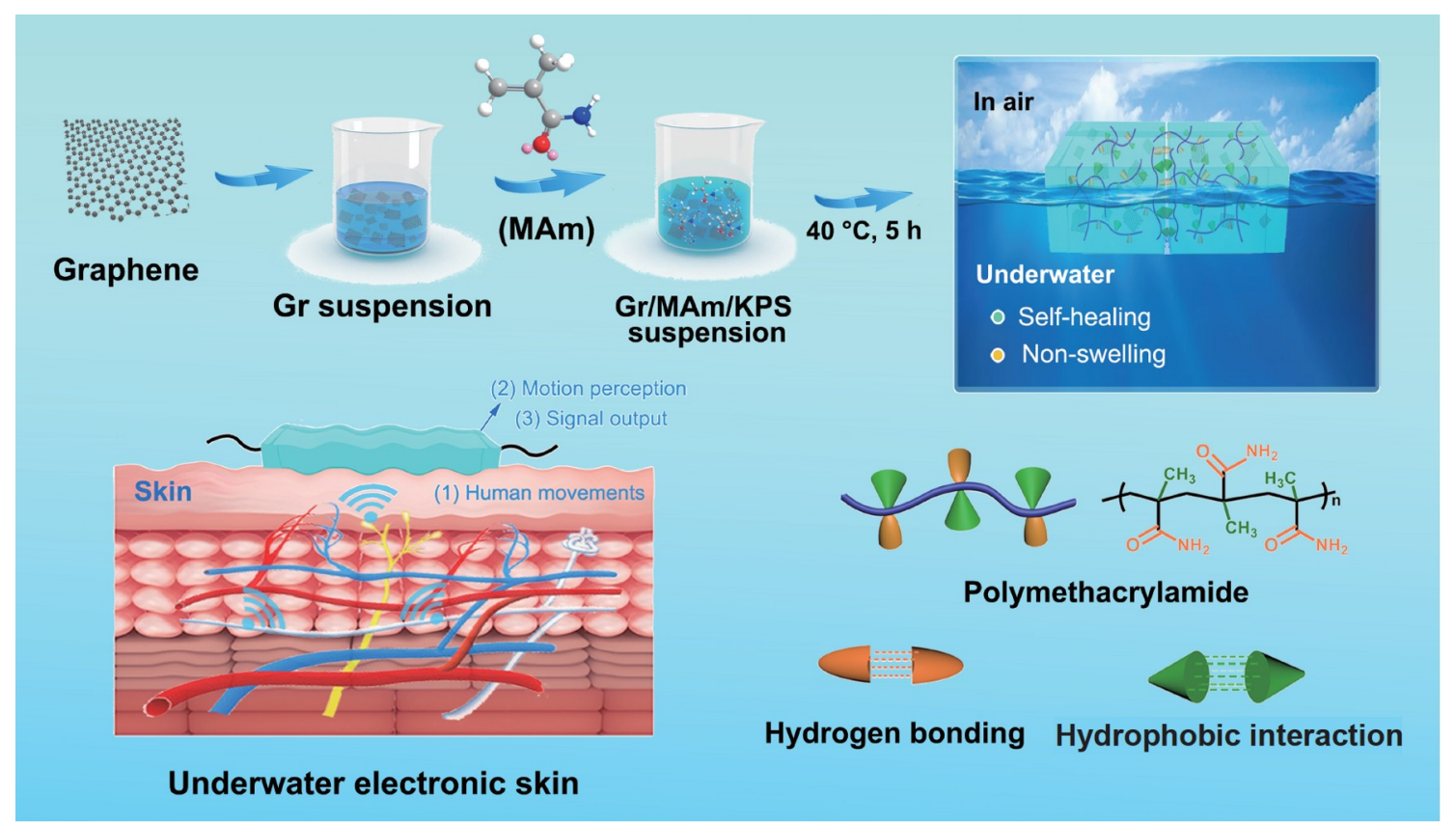

Figure 1 The fabrication process of underwater self-healing and anti-swelling hydrogels for underwater flexible electronic skin. 
aqueous environments. As displayed in Fig. 2a, PMAm hydrogel presented an excellent non-swelling property in various aquatic environments, including water, seawater, sweat, $\mathrm{pH} 11$ and $\mathrm{pH} 3$ aqueous solutions, and the corresponding SRs were all below 0.2 , which were significantly less than that of most traditional hydrogels. The prominent non-swelling performance was attributed to the water-resistance from hydrophilic and hydrophobic synergism. To verify the contribution of synergistic hydrophilicity and hydrophobicity on the non-swelling feature, the swelling property of PAm hydrogel was further comparatively evaluated. As expected, the PAm hydrogel exhibited an obvious structural collapse after swelling for $24 \mathrm{~h}$ (Fig. 2a) and demonstrated a significant SR of 1.2 (Fig. 2b). Besides, the interacting ability of water molecules on polymers significantly affected the waterresistance of hydrogels. TGA was thus performed on both PMAm and PAm hydrogels. As shown in Fig. 2c, the temperature at which "bound water" disappeared from PMAm hydrogel was lower than that for PAm hydrogel, suggesting the weak interaction between water molecules and PMAm. These results confirm the water-resistant ability originating from the methyl groups of PMAm.

Mechanical performances and self-healing were significant for the application of hydrogels in wearable and flexible electronics. As demonstrated in Fig. 3a, the tensile performance of hydrogels was investigated. The introduction of $\mathrm{Gr}$ significantly improved the tensile strength, fracture strain, toughness, and elastic modulus due to the Gr-assisted energy dissipation during the molecular chain motion. The mechanical performances of Gr-PMAm-2.8 hydrogel with Gr were obviously higher than that of PMAm-2.8 hydrogel. Moreover, the tensile strength, toughness, and elastic modulus of the hydrogels were enhanced by the increasing content of MAm because of the improved molecular network density and molecular interaction (Fig. S3). The tensile toughness presented a decline with an excessive rise of MAm, because the continuously increasing molecular interaction limited the movement of the polymer chains.

Furthermore, the recovery was explored through the rheological behavior of hydrogels. The hydrogel exhibited a typical solid behavior, and the gel transition point was determined at a strain of $16 \%$ (Fig. 3b). The cyclic stepstrain oscillatory sweep between small $(0.1 \%)$ and large strain $(50 \%)$ was performed on the hydrogel. As shown in Fig. 3c, the hydrogel exhibited a gel-like elastic behavior at a small strain sweep. On the contrary, the elastic network of the hydrogel was significantly damaged under a large strain sweep. However, the storage modulus $\left(G^{\prime}\right)$ and loss modulus $\left(G^{\prime \prime}\right)$ of the hydrogel could immediately return to the original level once the oscillatory sweep was adjusted to the strain of $0.1 \%$.

The underwater self-healing capability was a highly desirable feature for improving the reliability and durability of hydrogel electronic applications in wet or aquatic environments, such as sweaty skin, rainy weather, and underwater. As displayed in Fig. 3d, the hydrogel demonstrated a brilliant underwater self-healing performance. The whole underwater healing process of hydrogel was demonstrated in Movie S1. The hydrogel sample was firstly cut off by a pair of scissors, and then two fractured segments were placed in water and contacted under gentle squeeze only for $8 \mathrm{~s}$. Subsequently, the healed hydrogel was subjected to an extensive stretched deformation in water, and the healed interface can successfully retain excellent integrity without any damage and breakage. To confirm the contribution of the methyl group on underwater self-healing of PMAm hydrogel, the underwater healing performance of PAm hydrogel was also explored for comparison. As shown in Movie S2, the PAm hydrogel cannot achieve the self-healing performance in water due to the high hydrophilicity of polymer networks. To further assess the self-healing performance
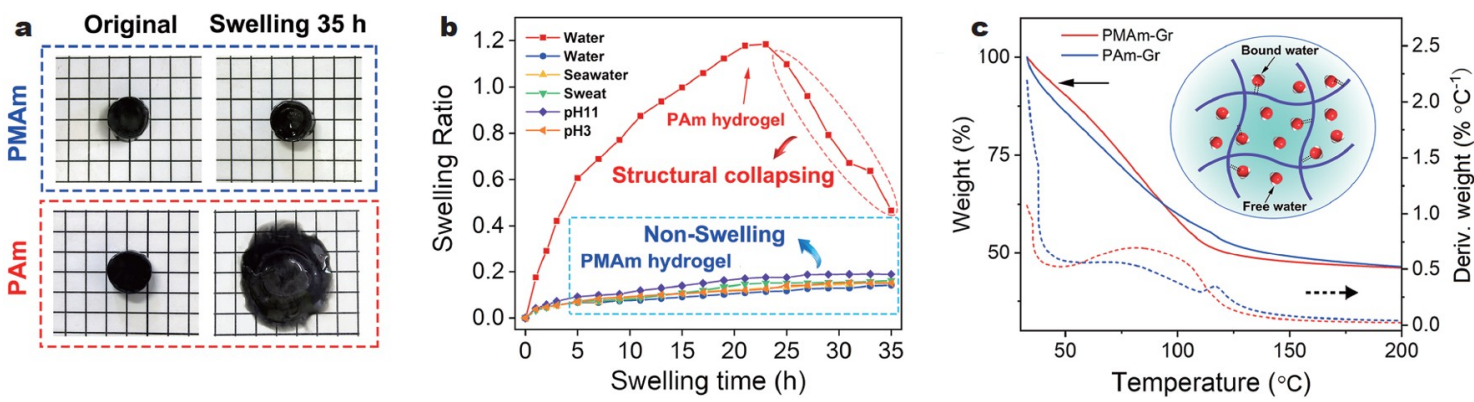

Figure 2 Swelling performance of Gr-PMAm-3.4 and Gr-PAm-3.4 hydrogels: (a) swelling in deionized water; (b) swelling curves of hydrogels in different aqueous solutions. (c) TGA curves of Gr-PMAm-3.4 and Gr-PAm-3.4 hydrogels. Inset: schematic diagram of "free water" and "bound water" in hydrogel networks. 

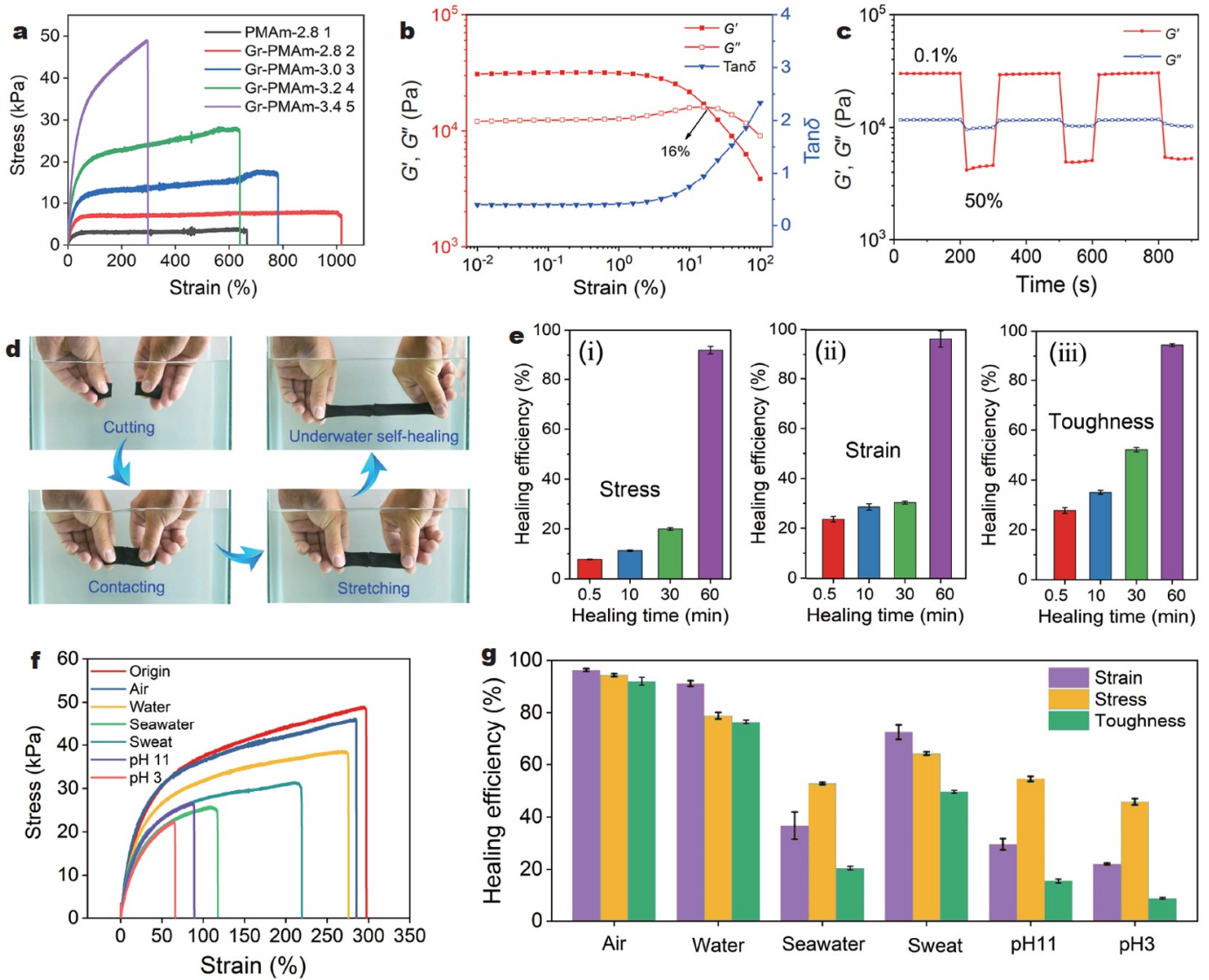

Figure 3 (a) Tensile property of hydrogels with different components. Rheological behavior of Gr-PMAm-3.4 hydrogel: (b) by dynamic strain sweep and (c) by cyclic step-strain oscillatory sweep between $0.1 \%$ and $50 \%$ strain. (d) Underwater self-healing of Gr-PMAm-3.4 hydrogel. (e) Healing efficiency for tensile stress, break strain, and toughness of Gr-PMAm-3.4 hydrogel for different healing times. (f) Tensile curves and (g) the corresponding tensile healing efficiency of Gr-PMAm-3.4 hydrogel healed in diverse aqueous solution environments for 60 min.

of the hydrogels, the mechanical performances of the healed hydrogels were carried out. The hydrogel exhibited a time-dependent cumulative healing phenomenon. The mechanical healing efficiency gradually improved with time, and the healing efficiency of stress, strain, and toughness can reach $96.36 \%, 94.42 \%$, and $92.03 \%$ after healing for $60 \mathrm{~min}$, respectively (Fig. 3e and Fig. S4). As shown in Fig. 3f, the hydrogel possessed brilliant selfhealing in diverse media environments, including air, water, seawater, sweat, alkali and acidic aqueous solutions. The corresponding mechanical healing efficiency was demonstrated in Fig. 3g. The different healing capability was attributed to the different contributions and influence of the physical healing interaction from the medium molecules, such as water, inorganic salts, base, and acid molecules.

As shown in Fig. 4a, the hydrogel possessed a brilliant electromechanical response capability based on the Gr- driven conduction mechanism, and the relative resistance change presented an almost linear growth with the increase of strain. To precisely evaluate the sensing ability, the gauge factors (GF) of segmented strain were calculated by the ratio of relative resistance change to strain. The hydrogel demonstrated high sensitivity in the whole strain region from $0 \%$ to $50 \%$ (Fig. 4a). The cyclic strain sensing of hydrogel under different strains was performed to evaluate their stability and reliability. The hydrogel sensors can achieve a continuous and real-time resistance variation for sensing different strains (Fig. 4b). The hydrogel also demonstrated a nimble response capability of strain sensing by accurately distinguishing different stretching frequencies (Fig. 4c). The results confirmed the excellent network resilience of the hydrogel and suggested a promising potential in constructing electrically and mechanically self-healing functions.

Electrical self-healing performance was also much de- 

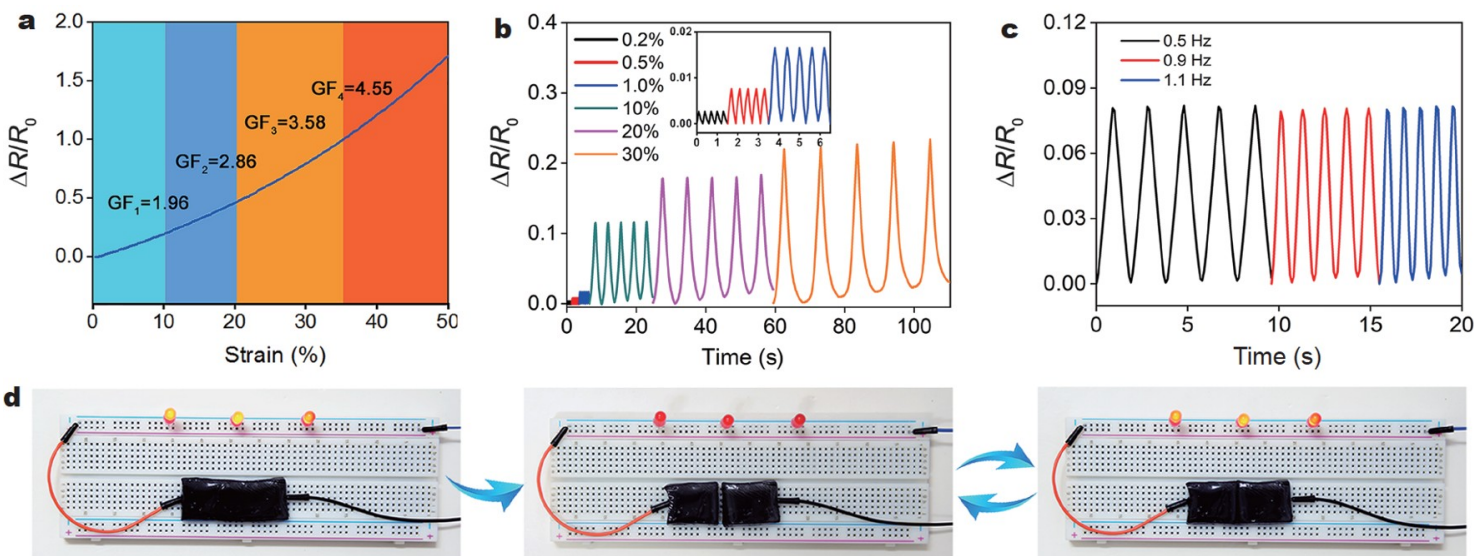

Original
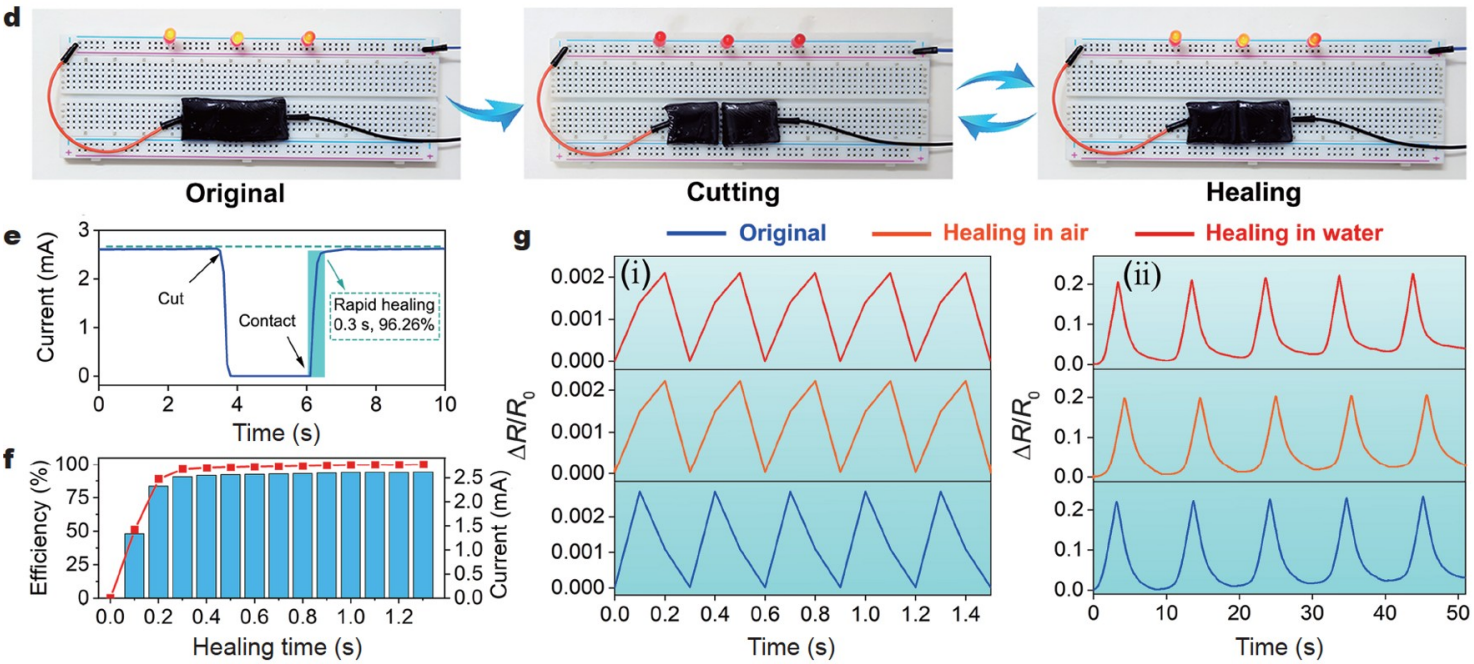

Healing

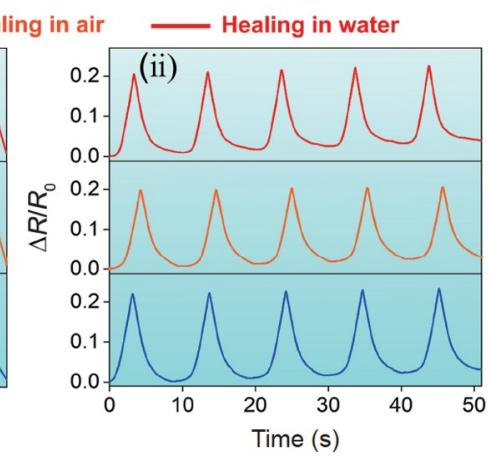

Figure 4 Resistance variation of Gr-PMAm-3.4 hydrogel: (a) with the increase of strain; (b) under diverse strain levels from 0.2\% to 30\% (inset: small strain of $0.2 \%-1 \%$ ); (c) under diverse tensile frequencies at 5\% strain. (d) Exhibition of self-healing conductive performance of Gr-PMAm-3.4 hydrogel. (e, f) Electrical healing and the corresponding conductive healing efficiency of Gr-PMAm-3.4 hydrogel with time evolution. The process mainly includes original state, cutting, re-contacting, and healable state. (g) Comparison of strain sensing performances for Gr-PMAm-3.4 hydrogel healed in air and water ((i) $0.2 \%$ and (ii) $30 \%$ strain sensing).

sired for the construction of flexible electronics. Fig. $4 \mathrm{~d}$ depicts the excellent electronic self-healing of hydrogel, and the real-time healing current curve and efficiency of the hydrogel are shown in Fig. 4e, f. The hydrogel demonstrated a fast electronic healing speed. Furthermore, as shown in Fig. 4g, the hydrogel healed in air and water both can achieve stable and accurate strain sensing of a wide strain range without any performance degradation. The excellent electronic and mechanical self-healing performance in diverse aquatic environments would provide enormous promising opportunities for durable underwater electronics.

Taking advantage of the non-swelling and underwater self-healing properties, the hydrogels provide a promising advance for underwater mechanical sensing. As displayed in Fig. 5a, the brightness of the diode decreased as the tensile strain of hydrogels increased. The underwater sensing ability of the hydrogels was further tested by the testing system demonstrated in Fig. 5b. The corresponding mechanical movements (including stretching, pressuring, and bending movements) were carried out in water, and the underwater mechanical deformation was further monitored by the change of resistance signal. As displayed in Fig. $5 c$, the hydrogel sensors demonstrated a stable and reliable underwater sensing capability for various mechanical stimulations. The mechanical bending, stretching, and pressuring stress were accurately monitored and perceived through stable electromechanical signal conversion.

Human skin is an self-healable organ equipped with ultrasensitive mechanoreceptors for feeling various external stimuli. Equally, the accurate perception of dynamic stimuli is also greatly desirable for electronic skin. As shown in Fig. 5d, a dynamic shear force was repeatedly performed on the surface of hydrogel sensors. The realtime stimulus signal variation was clearly recorded and differentiated by the change of relative resistance of sensors. At first, the contact pressure of the soft brush on the surface enabled the resistance with a noticeable increase, and then the resistance variation gradually de- 
a

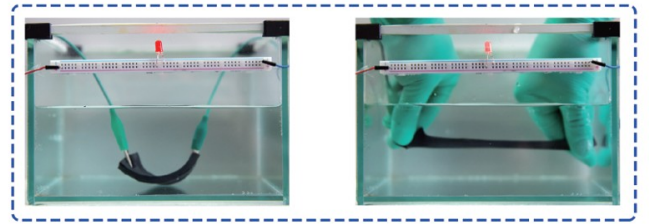

b

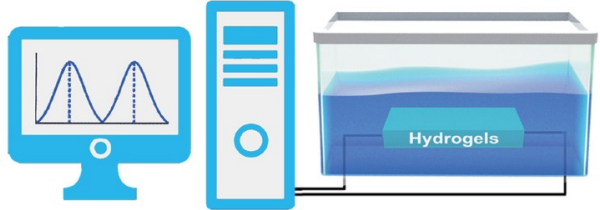

c

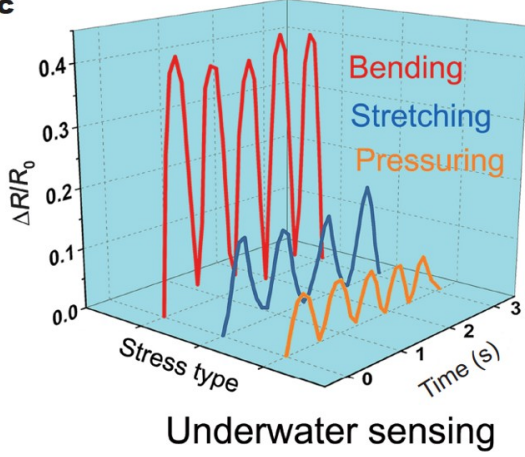

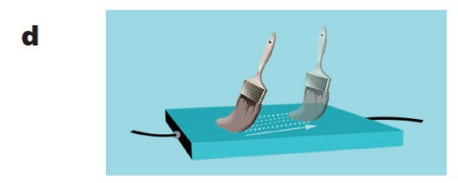

e
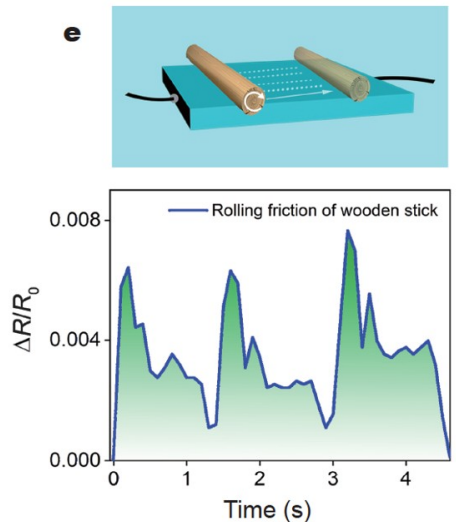

$\mathbf{f}$
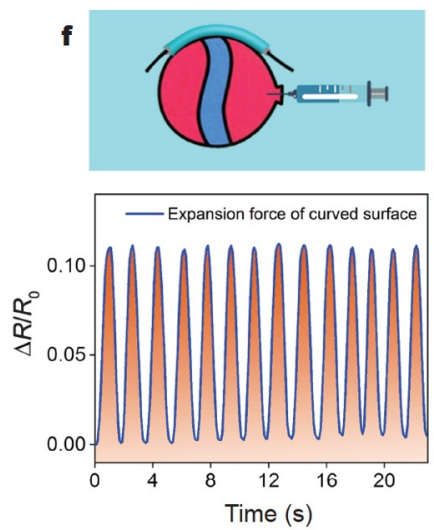

Figure 5 (a) Underwater sensing of hydrogel for stretch stress. The exhibition system of underwater sensing includes the hydrogel sensor, power supply, diode, wire, and sink. (b) Illustration of the hydrogel underwater sensing testing system consisting of an electrochemical workstation and a computer. The electrochemical workstation can accurately record the resistance changes of hydrogel sensors under diverse mechanical deformations in the sink. (c) The resistance variation of hydrogel sensors for monitoring continuous and repetitive mechanical movements in the water by the underwater sensing testing system, including bending, stretching, and pressuring. Each resistance variation curve respectively demonstrates a mechanical sensing signal change. Mechanical perception of hydrogel sensors for dynamic stress stimulus in the air: the resistance variation of GrPMAm-3.4 hydrogel sensors (d) for shear force by sliding a soft brush, (e) for rolling friction by a wooden stick, and (f) for expansion force of a curved balloon surface (plane sketch).

clined with the reduction of contact friction during brush sliding across the hydrogel surface. The hydrogel sensors can accurately detect the difference in the contact pressure and sliding strength during each slide. Moreover, the cyclic rolling friction of a wooden stick can also be continuously monitored and reflected by the real-time resistance signal change (Fig. 5e). Fig. 5f depicts the curved surface adaptability of hydrogel sensors. The hydrogel sensor was attached to the surface of a balloon connected with a syringe. When a certain amount of gas was repeatedly injected and withdrawn from the balloon, the volume of the balloon presented periodic expansion and contraction accordingly. The whole changing process was accurately monitored by the hydrogel sensors through continuous and uniform changes in resistance.

The prominent resolution and sensitivity of the selfhealing hydrogel sensor demonstrated tremendous potential in electronic skin. As displayed in Fig. 6a, the hydrogel sensors were assembled on the human finger through an adhesive tape. The changes in resistance signals accurately recorded the different bending angles of the finger. The hydrogel sensors can also achieve continuous and stable motion monitoring for wrist bending and leg bending (Fig 6b, c). Moreover, medium-amplitude movements of nodding, breathing, and chewing can also be perceived precisely (Fig. $6 \mathrm{~d}-\mathrm{f}$ ).

Furthermore, the monitoring of micro-movements from a human is key to electronic skin applications. As depicted in Fig. 6g, the artificial throat as a model of electronic skin was selected to evaluate the self-healing hydrogel for human health diagnosis and monitoring of physiological movements. As expected, the hydrogel sensors can feel and monitor the vibration of the throat when coughing. Moreover, the action of drinking water was wholly detected and reflected by a continuously discernible change in the resistance signal. The sensors 

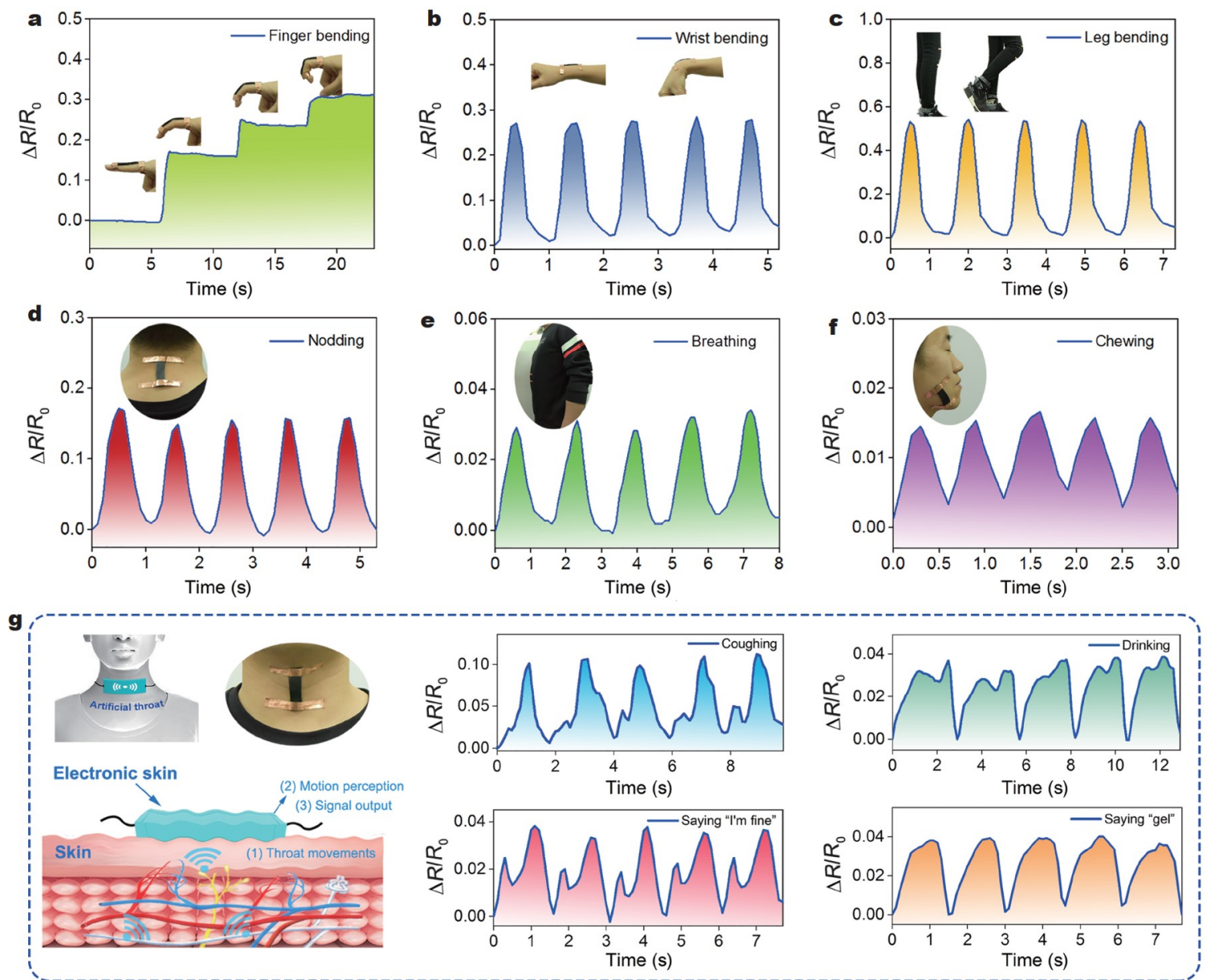

Figure 6 Resistance variation of underwater electronic skin for monitoring the human motions: (a) finger bending, (b) wrist bending, (c) leg bending, (d) nodding, (e) breathing, and (f) chewing (inset: the display of corresponding human body test parts detected by the hydrogel sensors). (g) Schematic illustration of the artificial throat on human skin for human motion perception (left); resistance variation of the artificial throat for monitoring human motions: coughing, drinking, saying "I'm fine" and "gel" (right). The specific test was carried out on Gr-PMAm-3.4 hydrogel.

could also precisely monitor and distinguish the difference of pronunciation signal when saying "I'm fine" and "gel".

\section{CONCLUSION}

In summary, flexible electronics have gained considerable interest in promising applications in aquatic and biological environments, yet achieving a self-healable hydrogel electronic in water is challenging. In this investigation, we fabricated a tough and conductive self-healing hydrogel composed of PMAm and Gr flakes. The hydrogel presented prominent water-insensitive self-healing properties in various aqueous solutions including deionized water, seawater, sweat, highly basic and acidic aqueous solutions. The underwater self-healing originated from hydrophobic interaction, hydrogen bonding, and chain diffusion of the hydrophilic and hydrophobic supramo- lecular network. Moreover, the self-healing hydrogel also possessed prominent mechanical toughness, high stretchability, and electromechanical properties and was designed as an electronic skin featuring fast responsiveness, reliability, and high sensitivity for detecting breathing, saying, coughing, and diverse body movements. The strategy of underwater self-healing hydrogel can empower considerable inspiration for designing future self-healing materials in aquatic environments. It is anticipated that water-insensitive self-healing hydrogel electronics would initiate great potential for promoting the development of bioelectronics, electronic skins, human-machine interface, and implantable devices.

Received 3 March 2021; accepted 23 April 2021; published online 2 July 2021

1 Kim HJ, Chen B, Suo Z, et al. Ionoelastomer junctions between 
polymer networks of fixed anions and cations. Science, 2020, 367: 773-776

2 Yuk H, Lu B, Zhao X. Hydrogel bioelectronics. Chem Soc Rev, 2019, 48: 1642-1667

3 Choi S, Han SI, Jung D, et al. Highly conductive, stretchable and biocompatible $\mathrm{Ag}$ - $\mathrm{Au}$ core-sheath nanowire composite for wearable and implantable bioelectronics. Nat Nanotech, 2018, 13: 10481056

4 Zhu M, Sun Z, Zhang Z, et al. Haptic-feedback smart glove as a creative human-machine interface (HMI) for virtual/augmented reality applications. Sci Adv, 2020, 6: eaaz8693

5 Huang $\mathrm{Z}$, Hao $\mathrm{Y}$, Li Y, et al. Three-dimensional integrated stretchable electronics. Nat Electron, 2018, 1: 473-480

6 Jo M, Min K, Roy B, et al. Protein-based electronic skin akin to biological tissues. ACS Nano, 2018, 12: 5637-5645

7 Lei Z, Huang J, Wu P. Traditional dough in the era of internet of things: Edible, renewable, and reconfigurable skin-like iontronics. Adv Funct Mater, 2020, 30: 1908018

8 Wang Q, Ling S, Liang X, et al. Self-healable multifunctional electronic tattoos based on silk and graphene. Adv Funct Mater, 2019, 29: 1808695

9 Liao M, Wan P, Wen J, et al. Wearable, healable, and adhesive epidermal sensors assembled from mussel-inspired conductive hybrid hydrogel framework. Adv Funct Mater, 2017, 27: 1703852

10 Li C, Iscen A, Sai H, et al. Supramolecular-covalent hybrid polymers for light-activated mechanical actuation. Nat Mater, 2020, 19: 900-909

11 Pal A, Goswami D, Martinez RV. Elastic energy storage enables rapid and programmable actuation in soft machines. Adv Funct Mater, 2020, 30: 1906603

12 Huang $\mathrm{Y}$, Zhong $\mathrm{M}$, Shi F, et al. An intrinsically stretchable and compressible supercapacitor containing a polyacrylamide hydrogel electrolyte. Angew Chem Int Ed, 2017, 56: 9141-9145

13 Rong Q, Lei W, Huang J, et al. Low temperature tolerant organohydrogel electrolytes for flexible solid-state supercapacitors. Adv Energy Mater, 2018, 8: 1801967

14 Zhang YS, Khademhosseini A. Advances in engineering hydrogels. Science, 2017, 356: eaaf3627

15 Liu X, Zhang Q, Gao G. DNA-inspired anti-freezing wet-adhesion and tough hydrogel for sweaty skin sensor. Chem Eng J, 2020, 394: 124898

16 Han L, Liu K, Wang M, et al. Mussel-inspired adhesive and conductive hydrogel with long-lasting moisture and extreme temperature tolerance. Adv Funct Mater, 2018, 28: 1704195

17 Kang J, Tok JBH, Bao Z. Self-healing soft electronics. Nat Electron, 2019, 2: 144-150

18 Greca LG, Lehtonen J, Tardy BL, et al. Biofabrication of multifunctional nanocellulosic 3D structures: A facile and customizable route. Mater Horiz, 2018, 5: 408-415

19 Huynh T-, Khatib M, Haick H. Self-healable materials for underwater applications. Adv Mater Technol, 2019, 4: 1900081

20 Ahn BK, Lee DW, Israelachvili JN, et al. Surface-initiated selfhealing of polymers in aqueous media. Nat Mater, 2014, 13: 867872

21 Kamata H, Akagi Y, Kayasuga-Kariya Y, et al. "Nonswellable" hydrogel without mechanical hysteresis. Science, 2014, 343: 873875

22 Kong W, Li T, Chen C, et al. Strong, water-stable ionic cable from bio-hydrogel. Chem Mater, 2019, 31: 9288-9294

23 Li J, Celiz AD, Yang J, et al. Tough adhesives for diverse wet surfaces. Science, 2017, 357: 378-381

24 Liu X, Zhang Q, Duan L, et al. Tough adhesion of nucleobasetackifed gels in diverse solvents. Adv Funct Mater, 2019, 29: 1900450

25 Bai S, Zhang X, Lv X, et al. Bioinspired mineral-organic bone adhesives for stable fracture fixation and accelerated bone regeneration. Adv Funct Mater, 2020, 30: 1908381

26 Cao Y, Wu H, Allec SI, et al. A highly stretchy, transparent elastomer with the capability to automatically self-heal underwater. Adv Mater, 2018, 30: 1804602

27 Kang J, Son D, Wang GJN, et al. Tough and water-insensitive selfhealing elastomer for robust electronic skin. Adv Mater, 2018, 30: 1706846

28 Hummer G, Garde S, García AE, et al. The pressure dependence of hydrophobic interactions is consistent with the observed pressure denaturation of proteins. Proc Natl Acad Sci USA, 1998, 95: 15521555

29 Liu X, Zhang Q, Gao G. Solvent-resistant and nonswellable hydrogel conductor toward mechanical perception in diverse liquid media. ACS Nano, 2020, 14: 13709-13717

30 Wang S, Urban MW. Self-healing polymers. Nat Rev Mater, 2020, 5: $562-583$

31 Gross M, Jaenicke R. Proteins under pressure. The influence of high hydrostatic pressure on structure, function and assembly of proteins and protein complexes. Eur J Biochem, 1994, 221: 617630

32 Wang $\mathrm{C}, \mathrm{Hu} \mathrm{K}$, Zhao C, et al. Customization of conductive elastomer based on PVA/PEI for stretchable sensors. Small, 2020, 16: 1904758

Acknowledgements This work was supported by the National Natural Science Foundation of China (51873024) and the grant from Science and Technology Department of Jilin Province (20200708102YY).

Author contributions Liu X designed the research ideas and wrote the article. Liu $\mathrm{X}$ and Zhang $\mathrm{Q}$ completed the data analysis of the experiment. Jia $\mathrm{F}$ and Gao G revised and corrected the manuscript.

Conflict of interest The authors declare that they have no conflict of interest.

Supplementary information Supporting data are available in the online version of the paper.

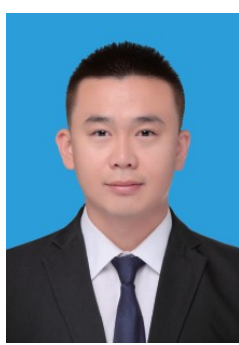

Xin Liu is currently a $\mathrm{PhD}$ candidate in the Polymeric and Soft Materials Laboratory at the School of Chemical Engineering, Changchun University of Technology. His current research focuses on the design of wet adhesive hydrogel materials as well as their applications for construction of bio-adhesion interface and humanmachine interfaces. 


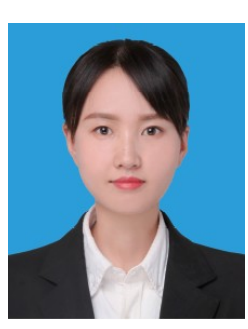

Qin Zhang is currently a $\mathrm{PhD}$ candidate in the Polymeric and Soft Materials Laboratory at the School of Chemical Engineering, Changchun University of Technology. Her current research focuses on the design and synthesis of smart hydrogels and their applications in humanmachine interfaces and flexible energy storage.

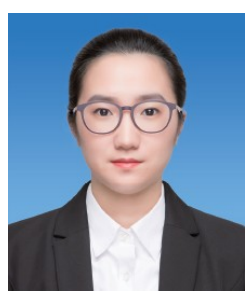

Fei Jia is an associate professor at the School of Chemical Engineering, Changchun University of Technology. She obtained her $\mathrm{PhD}$ degree from Jilin University. Her current research focuses on the application of polymeric hydrogels in medical dressings, flexible sensing, and energy storage.

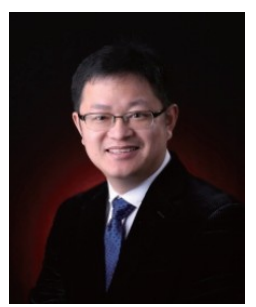

Guanghui Gao is a professor at the School of Chemical Engineering and Advanced Institute of Materials Science of Changchun University of Technology. He obtained his bachelor's degree from Shanghai Jiao Tong University and $\mathrm{PhD}$ degree from Sungkyunkwan University, Korea. $\mathrm{He}$ founded the Polymeric and Soft Materials Laboratory at Changchun University of Technology. His current research focuses on the application of polymer hydrogels in medical dressings, flexible electronics, and energy storage.

\section{抗溶胀自愈合水凝胶构筑水下柔性机械感受器}

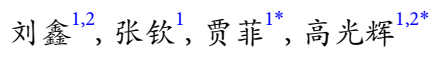

摘要 近年来, 具有抗湿功能的柔性电子器件在湿润环境如汗湿的 皮肤、雨水天气以及生物体液环境等方面的应用获得了越来越多 的关注. 然而, 设计出一种具有非溶胀且水下自愈合性的水凝胶材 料用以实现其在水溶液环境下稳定的机械感知至今仍是个巨大的 挑战. 本文中, 我们成功构建了一种抗溶胀自愈合水凝胶, 其在各种 水溶液环境(包括水、海水、汗水、酸碱水溶液等)中展示出优异 的自愈合行为. 此外, 该水凝胶展现出高的拉伸性以及水下机械传 感性能. 由该水凝胶进一步装备而成的可水下自愈合的电子皮肤 具有响应速度快、可靠性好、灵敏度高等特点, 并成功用于准确 监测人体呼吸、说话、肢体运动等. 该水凝胶传感器在水中先断 裂再愈合之后, 仍然保持着优异的水下机械传感性能. 这种水下自 愈合且抗溶胀的水凝胶为制备水生环境下的各种稳定电子器件如 水下机器人、可植入电子器件, 以及实现水下通讯等提供了可能. 